\title{
Predictors of change in the peripheral skeletons of older adults in rural Gambia: The Gambian bone and muscle ageing study
}

\author{
M. Ó Breasail ${ }^{1}$, C. M. Parsons ${ }^{2}$, A. Zengin ${ }^{3}$, L. M. A. Jarjou ${ }^{4}$, A. Prentice ${ }^{1,4}$, C. Cooper ${ }^{2}$ and \\ K. A. Ward ${ }^{2,4}$ \\ ${ }^{1}$ MRC Nutrition and Bone Health Research Group, Cambridge, UK, \\ ${ }^{2}$ MRC Lifecourse Epidemiology, Human Development \& Health, University of Southampton, Southampton, UK, \\ ${ }^{3}$ Department of Medicine, School of Clinical Sciences at Monash Health, Monash University, Australia and \\ ${ }^{4}$ MRC Unit The Gambia, London School of Hygiene and Tropical Medicine, Banjul, The Gambia
}

Nutritional and epidemiological transition resulting from rapid urbanization is occurring across sub-Saharan Africa (SSA). Coupled with increasing longevity, the incidence of osteoporosis and other chronic musculoskeletal conditions are expected to rise greatly(1,2). No longitudinal studies to date describe musculoskeletal ageing in SSA and data are urgently needed to inform policy. The aim was to explore baseline predictors of skeletal change in a longitudinal study of musculoskeletal ageing in rural Gambia using peripheral quantitative computed tomography $(\mathrm{pQCT})^{(3)}$.

pQCT scans were acquired at the non-dominant radius (at $4 \%, 33 \%$ of radius length) of participants ( $\mathrm{n}=322,52.5 \%$ women) aged 40-92 (mean (SD) 60(12)) years at baseline. Outcomes were total volumetric bone mineral density (vBMD), trabecular vBMD, total area, bone mineral content (BMC), cortical vBMD, and total area 33\%. Potential predictors investigated were: 1) bone turnover and related markers (serum collagen type 1 cross-linked $\beta$-C-telopeptide (CTX), type 1 procollagen N-terminal (P1NP), parathyroid hormone $(\mathrm{PTH}), 25(\mathrm{OH}) \mathrm{D}), 2$ ) muscle function measures (handgrip dynamometry (max grip, N/kg), jumping mechanography (relative force $(\mathrm{kN} / \mathrm{kg}$ ) and relative power $(\mathrm{kW} / \mathrm{kg})$ ), 3) body composition (fat mass $(\mathrm{FM})$ and appendicular lean mass (ALM) residuals). Annualised pQCT change was calculated, and multiple regression used to explore predictors of bone change within sex (M, F), models were adjusted for baseline bone values, and output presented as mean change $[95 \% \mathrm{CI}]$.

Annualised decreases in total vBMD (M -1.3 (3.48)\%, F -1.70(3.07)\%, BMC (M - $0.59(1.49) \%, \mathrm{~F}-1.16(2.30) \%)$ were observed but trabecular loss only in women (-1.06(4.62)\%). Distal area increased (M 1.13(4.32)\%, F 0.72(3.77)\%), though proximal area decreased $(\mathrm{M}-0.74(2.52) \%, \mathrm{~F}-0.47(2.52) \%)$. Age was a negative predictor of change in total vBMD $\left(\mathrm{M}-0.08[-0.14,-0.03]^{0} \%, \mathrm{~F}-0.06\right.$ $[-0.11,-0.01] \%)$, trabecular vBMD (F-0.12[-0.19,-0.04]\%), cortical vBMD (F -0.03[-0.05,-0.01]\%) and BMC (M -0.02[-0.05,-0.01]\%) but a positive predictor of $4 \%$ total area (M $0.08[0.01,0.14] \%)$. In men, ALM residuals positively predicted total vBMD $(0.29$ $[0.04,0.53] \%$ ) change, and handgrip positively predicted change in total area (M 0.007[0.002,0.012]\%). In women, max relative force was a positive predictor of total $\operatorname{vBMD}(0.21[0.02,0.40] \%)$ but a negative predictor of distal area $(-0.22[-0.43,-0.01] \%)$. Max relative power was a positively associated with trabecular vBMD (F 0.17[0.03,0.32]\%), total vBMD (M 0.06 [0.01,0.12]\%) and BMC (M $\left.0.03[0.01,0.06]^{\%}\right)$. In women, BTMs were negatively associated with trabecular vBMD (CTX $-4.84[-7.96,-1.72]^{\%}, \mathrm{P} 1 \mathrm{NP}-2.50 \%$ $[-4.19,-0.82])$. In men, P1NP was negatively associated with BMC, cortical vBMD and total area 33\% $(-0.79[-1.54,-0.05] \%,-0.67$ $[-1.21,-0.13] \%$ and $1.32[0.11,2.48] \%)$

Advancing age was the main predictor of skeletal change in rural Gambians. The magnitude of change seen in this population was broadly in keeping with studies from high-income countries. In women, CTX and P1NP were strongly associated with decreases in trabecular vBMD. While better muscle function was associated with better bone outcomes, no consistent pattern was found. These data provide important insights into musculoskeletal ageing in SSA.

\section{References}

1. Gregson, C.L., et al. (2019) Lancet Glob Health, 7(1): p. e26-e27.

2. Aboderin, I.A.G. and J.R. Beard (2015) Lancet, 385(9968): p. e9-e11.

3. Zengin, A., et al. (2017) Front Endocrinol, 8: p. 219. 